\title{
Are compliance constants ill-defined descriptors for weak interactions?
}

Cite this: DOI: $10.1039 / \mathrm{c3ra22866e}$

\author{
Jörg Grunenberg*a and Giampaolo Barone ${ }^{\mathrm{b}}$
}

Received 14th August 2012,

Accepted 4th February 2013

DOI: $10.1039 / c 3 r a 22866 e$

\begin{abstract}
Just as the potential energy can be written as a quadratic form in internal coordinates, so it can also be expanded in terms of generalized forces. The resulting coefficients are termed compliance constants. In this article, the suitability of compliance constants as non-covalent bond strength descriptors is studied (a) for a series of weakly bound hydrogen halide-rare gas complexes applying a configuration interaction theory, (b) for a double stranded DNA 4-mer using approximate density functional methods and finally (c) for a double stranded DNA 20-mer using empirical force fields. Our results challenge earlier studies, which concluded the inappropriateness of compliance constants as soft matter descriptors. The discrepancy may be ascribed, inter alia, to the application of an oversimplified potential function in these earlier studies, assuming a central forces approximation.
\end{abstract}

www.rsc.org/advances

results. ${ }^{3,17}$ Very recently, in an elegant series (see for example ${ }^{18}$ ) of papers, Cremer et al. have shown that the so called local mode force constants introduced in $1998^{19}$ amount exactly to (inverse) compliance constants introduced as early as $1947 .{ }^{20} \mathrm{It}$ is important to note that, as a second order tensor, the compliance matrix contains non-zero coupling elements. If this tensor property is ignored and/or oversimplified potential functions are used, problems concerning the interpretation of compliance constants may arise. ${ }^{21-24}$ The interpretation of exactly those coupling terms seems to have caused some troubles in the literature, ${ }^{18}$ culminating in the conclusion that "compliance constants are not useful diagnostics of the strength of weak interatomic interactions" at all. ${ }^{21,22}$

In order to verify, or falsify, this finding and to further elucidate the interpretation of compliance off-diagonal terms, the following study was split into two parts: in part one, we present detailed results on a series of rare gas-hydrogen iodide complexes comprising solely of van der Waals bonding. Full quantum chemical coupling between the valence coordinates is maintained, the approximation of central forces is not assumed. In part two of the study, the robustness of our results is cross-checked by analyzing the results of double stranded DNA 4-mer and 20-mer models, computed at the density functional level (DFT) including dispersion corrections and at the molecular mechanics (MM) level, respectively. Again, full coupling between the valence coordinates is maintained. That means, we again go beyond the approximation of central forces.

\section{Van der Waals complexes}

Institut für Organische Chemie, Technische Universität Braunschweig, Hagenring 30, 38106 Braunschweig, Deutschland.E-mail: Joerg.Grunenberg@tu-bs.de ${ }^{b}$ Dipartimento di Scienze e Tecnologie Biologiche, Chimiche e Farmaceutiche, Università di Palermo, Viale delle Scienze, Edificio 17, 90128 Palermo, Italy
Theoretical studies of van der Waals complexes in terms of their structure, internal dynamics, electric properties, and 
photochemistry are a vivid field of research by itself, if nothing else because of their importance in soft matter research in general. ${ }^{25}$ Further, as prototypes of extremely soft hydrogen bonds, hydrogen halide-rare gas complexes are interesting from a biophysical viewpoint as well. As part of our ongoing effort to analyze and uniquely quantify soft, non-covalent interactions, ${ }^{9,26-29}$ we recently studied the complex between xenon atoms and the hydrogen halide HI. ${ }^{30}$ This very van der Waals molecule is particularly appealing due to the inherent maximal polarizability of xenon. Van der Waals forces arise from instantaneous charge fluctuations, and the static polarizability is therefore the single most important property responsible for this weak interaction. An increase of the rare gas polarizability should go hand in hand with a linear rise of the bonding force and comparison of the static polarizability with computed compliance constants should therefore allow for an independent assessment of the usability - or blurredness - of compliance constants as bond descriptors.

A general feature of $\mathrm{Rg}-\mathrm{HX}$ complexes is the existence of a pair of local potential minima separated by an orientational barrier. One minimum occurs when the hydrogen is located between the Rg and X atoms (the "hydrogen-bonded" Rg-HX isomer), another minimum is at the position of the hydrogen pointing away from $\mathrm{Rg}$ (denoted as the "anti-hydrogenbonded" $\mathrm{Rg}-\mathrm{XH}$ isomer). In the present study we focus on the "hydrogen-bonded" $\mathrm{Rg}-\mathrm{HX}$ isomers noting that the stabilities of the isomeric forms are nevertheless still under discussion. The stability order may even become reversed in the vibrational ground or first low-energy excited states of some complexes. ${ }^{31}$ Energies, geometry optimization and the energy second derivatives (Cartesian force constants) were computed at the quadratic configuration interaction including single and double excitation (QCISD) ${ }^{32}$ in order to systematically correct, at least in part, for the intrinsic errors in the Hartree-Fock method and density functional theory (DFT) when it comes to the description of long range dispersion. The frozen-core approximation for $\mathrm{Rg}$ and the iodine atoms was used throughout this study. An augmented correlation consistent basis set of triple zeta quality including polarization for hydrogen ${ }^{33}$ (aug-cc-pvtz) adding pseudopotentials for $\mathrm{Rg}^{34}$ and iodine $^{35}$ (aug-cc-pvtz-pp) was applied. Generalized compliance constants were calculated using our proposed procedure ${ }^{16,29}$ as implemented in the COMPLIANCE 3.0 code, which is freely available from our homepage. All electronic structure calculations were performed using the Gaussian 09 software suite ${ }^{36}$ and the atomic basis sets were taken from the EMSL database. $^{37}$

Table 1 and Fig. 1 compile the results of our compliance calculations for the series of $\mathrm{Rg} \cdot \cdots \mathrm{HI}$ complexes. The van der Waals interaction strength is spanning a range of more than one order of magnitude: starting with the extremely soft bonded $\mathbf{H e}$-.HI system showing a huge compliance constant of $93.37 \mathrm{~cm} \mathrm{~N}^{-1}$ (matching a relaxed force constant as weak as $0.010 \mathrm{~N} \mathrm{~cm}^{-1}$ ), the $\mathbf{R g} \cdots \mathbf{H I}$ interaction strength successively (Ne: $52.73 \mathrm{~cm} \mathrm{~N}^{-1}$; Ar: $30.31 \mathrm{~cm} \mathrm{~N}^{-1}$; $\mathrm{Kr}: 23.21 \mathrm{~cm} \mathrm{~N}^{-1}$ and Xe: $17.12 \mathrm{~cm} \mathrm{~N}^{-1}$ ) reaches $14.19 \mathrm{~cm} \mathrm{~N}^{-1}$, corresponding to a
Table 1 Theoretical compliance constants in $\mathrm{cm} \mathrm{N}^{-1}$ computed at the QCISD level of theory and experimental polarizabilities in a.u. for the series of $\mathrm{Rg} \cdots \mathrm{HI}$ van der Waals molecules

\begin{tabular}{lrrrrrr}
\hline & \multicolumn{1}{c}{$\mathrm{He}$} & \multicolumn{1}{c}{$\mathrm{Ne}$} & \multicolumn{1}{c}{$\mathrm{Ar}$} & \multicolumn{1}{c}{$\mathrm{Kr}$} & \multicolumn{1}{c}{$\mathrm{Xe}$} & \multicolumn{1}{c}{$\mathrm{Rn}$} \\
\hline$C_{\mathrm{RR}}$ & 93.37 & 52.73 & 30.31 & 23.21 & 17.12 & 14.19 \\
$C_{\mathrm{rr}}$ & 0.30 & 0.30 & 0.29 & 0.29 & 0.29 & 0.30 \\
$C_{\mathrm{xx}}$ & 93.90 & 53.21 & 31.01 & 23.50 & 17.51 & 14.25 \\
$C_{\mathrm{Rr}}$ & 93.49 & 52.82 & 30.77 & 23.33 & 17.33 & 14.07 \\
polarizability $^{46}$ & 1.38 & 2.67 & 11.07 & 17.07 & 27.8 & 33.18
\end{tabular}

relaxed force constant of $0.074 \mathrm{~N} \mathrm{~cm}^{-1}$ in the case of the

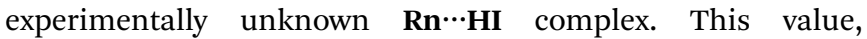
although purely dispersive, is already comparable to a weak classical hydrogen bond, ${ }^{26}$ and therefore (in terms of elasticity) closes the gap between pure induced-dipoleinduced-dipole and induced-dipole-permanent-dipole interactions. Since the static polarizability is the single most important property responsible for this weak interaction, an increase of the rare gas polarizability should therefore go hand in hand with a linear rise of the bonding force. Indeed, the softness of the van der Waals interaction, expressed as relaxed force constants, shows the expected linear decline $\left(C_{\mathrm{RR}}\right.$ in Table 1; see Fig. 1) as the rare gas polarizability rises, while the H-I covalent bond strength $\left(C_{\mathrm{rr}}\right.$ in Table 1$)$ is more or less unperturbed. We come to the following conclusion: using compliance constants as bond strength descriptors for soft interactions, the underlying physics is described qualitatively and quantitatively correctly.

What about the compliance coupling terms? First, we focus on the relaxed force constant $C_{\mathrm{xx}}$ connected with the coordinate $\mathbf{X}$, that means, the non-bonded distance $\mathbf{R g} \cdots \mathbf{I}$ (see Fig. 2). It was put forward ${ }^{22}$ that all non-bonded pairs of atoms should have a zero force constant (infinite compliance constant). ${ }^{38}$ To begin with, based on general curve sketching, zero force constants (second derivatives) may only come up for non-stationary points, namely at the inflection point of a

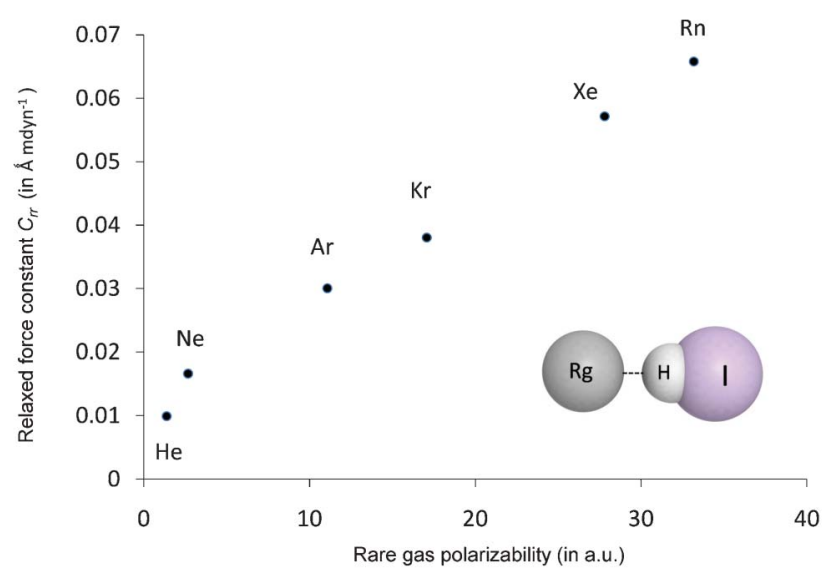

Fig. 1 The suitability of compliance constants as bond strength descriptors for weak interactions: a larger dispersion is connected with a stronger van der Waals bond expressed as relaxed force constant (inverse compliance constants). 


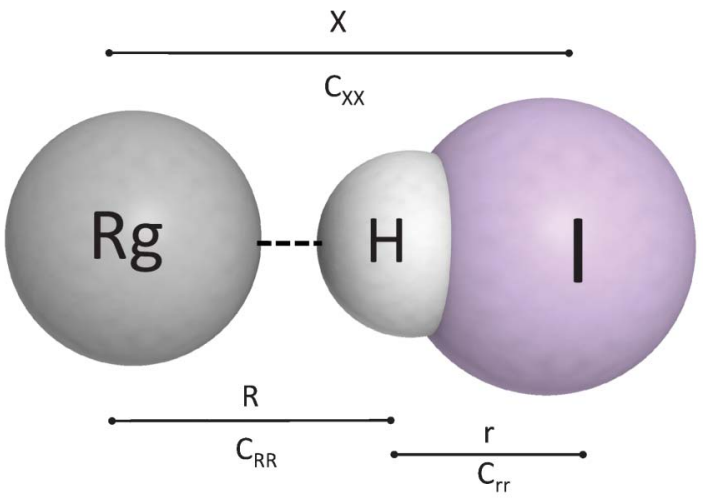

Fig. 2 Coordinate system for the rare gas $\mathrm{HI}$ complexes. The covalent (distance $\mathrm{H}-\mathrm{I}$ ) coordinate $r$ is represented by the relaxed force constant $\boldsymbol{C}_{\mathbf{r r}}$, the hydrogen bond coordinate $\boldsymbol{R}$ (distance $\mathbf{R g} \cdots \mathrm{H}$ ) by $\boldsymbol{C}_{\mathbf{R R}}$ and the non-bonded distance $\mathbf{R g} \cdots \mathbf{I}$ will be represented by the coordinate $\boldsymbol{X}$ and the corresponding relaxed force constant $\boldsymbol{C}_{\mathrm{xx}}$ respectively.

morse type energy curve. The emergence of a non-zero potential constant for non-neighbor atoms does therefore of course not preclude the use of compliance constants for the description of direct atom-atom interactions. This is also true for van der Waals contacts or hydrogen bonds. We now turn to the Taylor expansion of the potential energy. Taking a general quadratic potential as a mathematical basis, we arrive at the expression (1)

$$
2 V=C_{\mathrm{RR}} R^{2}+C_{\mathrm{rr}} r^{2}+C_{\mathrm{XX}} X^{2}+C_{\mathrm{rR}} r R+C_{\mathrm{rX}} r X+C_{\mathrm{RX}} R X
$$

where $V$ represents the potential energy, $C_{\mathrm{RR}}, C_{\mathrm{rr}}$ and $C_{\mathrm{Xx}}$ the diagonal terms (second derivatives), $C_{\mathrm{rR}}, C_{\mathrm{rX}}$ and $C_{\mathrm{RX}}$ the off diagonal terms (mixed second derivatives) according to the respective displacement. The potential function (1) is the underlying potential of all fully coupled quantum chemical force fields and it is therefore not surprising that due to our QCISD second derivative calculations all "through space" compliance constant $C_{\mathrm{xx}}$ display the same order of magnitude (see Table 1) in comparison with the relaxed force constant for the hydrogen bond coordinate $C_{\mathrm{RR}}$. In other words, the force induced displacement is more or less the same in both cases. The interpretation of the compliance coupling terms, which seem to have caused so many troubles in the literature, is then quite trivial: The off-diagonal elements describe the relaxation of all other internal coordinates in response to the applied external force. ${ }^{15,16}$ While according to (1) this coupling is visible through the coupling compliance constant $C_{\mathrm{fx}},{ }^{39}$ it is of course not transparent in a force field as applied by Pulay and Baker, assuming central forces (2):

$$
2 V=f R^{2}+F r^{2}
$$

In this approximation it is assumed that the forces holding the atoms in their equilibrium positions act only along the lines joining pairs of atoms. Pure ionic interactions are at work. In practice, this pre quantum approximation is of very limited use. ${ }^{40}$ It breaks down even in the case of $\mathrm{CO}_{2}{ }^{41}$ and, as we will show in the following, leads to erratic and contradicting predictions quite generally. Applying the approximate potential function (2) the "through space" force constant between $\mathbf{R g}$ and $\mathbf{I}$, that means $C_{\mathrm{XX}}$, should amount to

$$
C_{\mathrm{XX}}=C_{\mathrm{RR}} /\left(1+C_{\mathrm{RR}} / C_{\mathrm{rr}}\right)
$$

In the case of the rare gas HI complexes the prediction is authoritative: because the covalent bond between hydrogen and iodine $\left(C_{\mathrm{rr}}\right)$ is much stronger than the soft van der Waals interaction between the rare gas atom $\mathrm{Rg}$ and hydrogen $\left(C_{\mathrm{RR}}\right)$ (that is $C_{\mathrm{rr}} \gg C_{\mathrm{RR}}$ ), the "through space" relaxed force constant between $\mathbf{R g}$ and $\mathbf{I}\left(C_{\mathrm{xx}}\right)$ is comparable with the weak van der Waals bond between $\mathbf{R g} \cdot \cdot \mathbf{H I}$ for all noble gases (note the minimal coupling between $C_{\mathrm{RR}}$ and $C_{\mathrm{rr}}$ ), which is several orders of magnitude smaller. Nevertheless, in the case of symmetrical and coupled hydrogen bonds, for example present in the well studied $\mathrm{F}^{\cdots} \cdot \mathrm{HF}$ anion, ${ }^{42}$ both relaxed force constants, $C_{\mathrm{rr}}$ and $C_{\mathrm{RR}}$, are exactly the same. Applying again expression (2) the "through space" force constant between the two fluorine atoms $\left(C_{\mathrm{Xx}}\right)$ should amount to only $50 \%$ of the symmetrical hydrogen bond. Our calculated values however show that the actual $C_{\mathrm{Xx}}$ value for the $\mathrm{F}^{\cdots} \mathrm{HF}$ anion is nearly tripled in comparison with the hydrogen bond.

The compliance value of $0.44 \mathrm{~cm} \mathrm{~N}^{-1}$ may of course not be equated with a covalent bond between the two fluorine atoms in a Lewis sense nor with any calculated Mulliken bond order. It is just the measure of the strong force needed to compress two (strong) hydrogen bonds at once.

The breakdown of the central forces approximation and the dubiety of conclusions drawn from force field (2) as applied by Pulay and Baker are quite evident now: note the large (and in this case negative) coupling (Fig. 3), which is again not

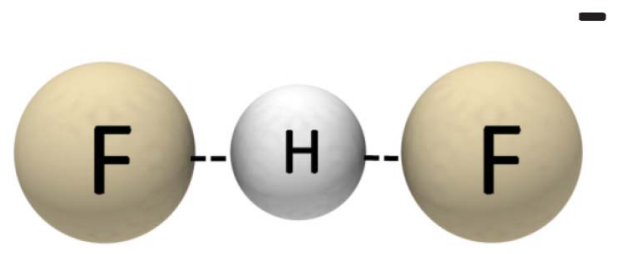

\begin{tabular}{|c|c|c|c|}
\hline & $F \ldots H F^{\prime}$ & $F H \ldots F^{\prime}$ & $F \ldots \ldots F^{\prime}$ \\
\hline$F \ldots H F^{\prime}$ & 1.18 & & \\
\hline$F H \ldots F^{\prime}$ & -0.96 & 1.18 & \\
\hline$F \ldots \ldots F^{\prime}$ & 0.21 & 0.21 & 0.44 \\
\hline
\end{tabular}

Fig. 3 Compliance matrix computed at the $\operatorname{CCSD}(\mathrm{T}) /$ aug-cc-pvqz level of theory (see text). The existence of the large through space compliance constant $F \cdots F^{\prime}$ $\left(0.44 \mathrm{~cm} \mathrm{~N}^{-1}\right)$ does not preclude the use of compliance constants for the description of the hydrogen bonds $\left(1.18 \mathrm{~cm} \mathrm{~N}^{-1}\right)$. Note the large (negative) coupling constant between the two hydrogen bond coordinates $\mathrm{F} \cdot \mathrm{HF}$ and $\mathrm{FH} \cdots \mathrm{F}^{\prime}$ of $-0.96 \mathrm{~cm} \mathrm{~N}^{-1}$, which is of the same order of magnitude as the diagonal terms. 
included in (2). Our computed compliance value of $1.18 \mathrm{~cm}$ $\mathrm{N}^{-1}$ (matching a relaxed force constant as strong as $0.84 \mathrm{~N}$ $\mathrm{cm}^{-1}$ ) for the $\mathrm{F}^{\cdots} \cdot \mathrm{H}$ bonds in the FHF anion is in line with the experimentally known and eye-catching short $R(\mathrm{~F} \cdots \mathrm{F})$ distance of $225 \mathrm{pm}^{42}$ The hydrogen bond in the FHF anion is well accepted as one of the strongest (if not the strongest) hydrogen bond in chemistry and the calculated compliance constant gives an account to this strength in a conclusive manner. Again, the existence of large through space relaxed force constants does not preclude the use of compliance constants for the description of the symmetrical hydrogen bonds themselves.
A

\begin{tabular}{|l|l|l|}
\hline 1 CG & $\mathrm{N}-\mathrm{H} \ldots \mathrm{O}$ & 4.03 \\
\cline { 2 - 3 } & $\mathrm{N} . . . \mathrm{H}-\mathrm{N}$ & 2.10 \\
\cline { 2 - 3 } & $\mathrm{O} \ldots \mathrm{H}-\mathrm{N}$ & 3.66 \\
\hline 2 AT & $\mathrm{C}-\mathrm{H} \ldots \mathrm{O}$ & 26.77 \\
\cline { 2 - 3 } & $\mathrm{N}-\mathrm{H} \ldots \mathrm{H}$ & 5.49 \\
\cline { 2 - 3 } & $\mathrm{N}-\mathrm{H} \ldots \mathrm{O}$ & 3.80 \\
\hline 3 CG & $\mathrm{N}-\mathrm{H} \ldots \mathrm{O}$ & 4.30 \\
\cline { 2 - 3 } & $\mathrm{N} . . . \mathrm{H}-\mathrm{N}$ & 2.01 \\
\cline { 2 - 3 } & $\mathrm{O} \ldots \mathrm{H}-\mathrm{N}$ & 3.23 \\
\hline 4 GC & $\mathrm{O} \ldots \mathrm{H}-\mathrm{N}$ & 4.01 \\
\cline { 2 - 3 } & $\mathrm{N}-\mathrm{H} \ldots \mathrm{N}$ & 2.17 \\
\cline { 2 - 3 } & $\mathrm{N}-\mathrm{H} \ldots \mathrm{O}$ & 3.73 \\
\hline
\end{tabular}

C

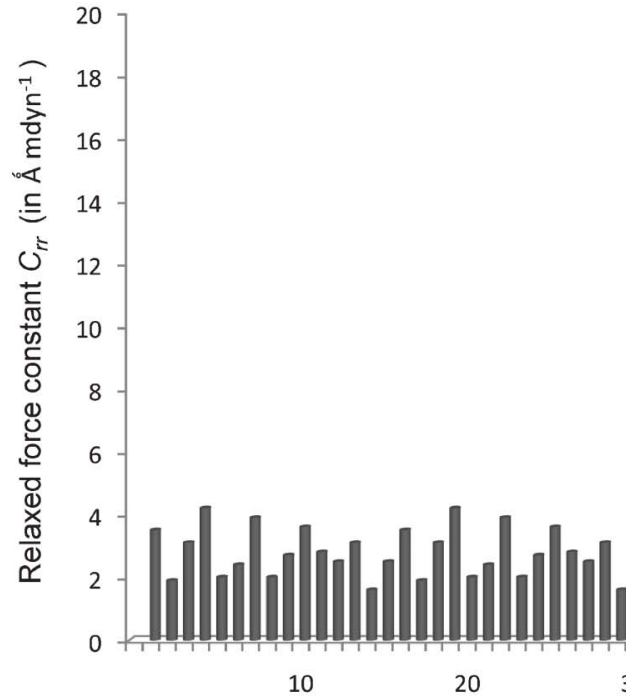

Base pair sequence of the model DNA 20-mer
B
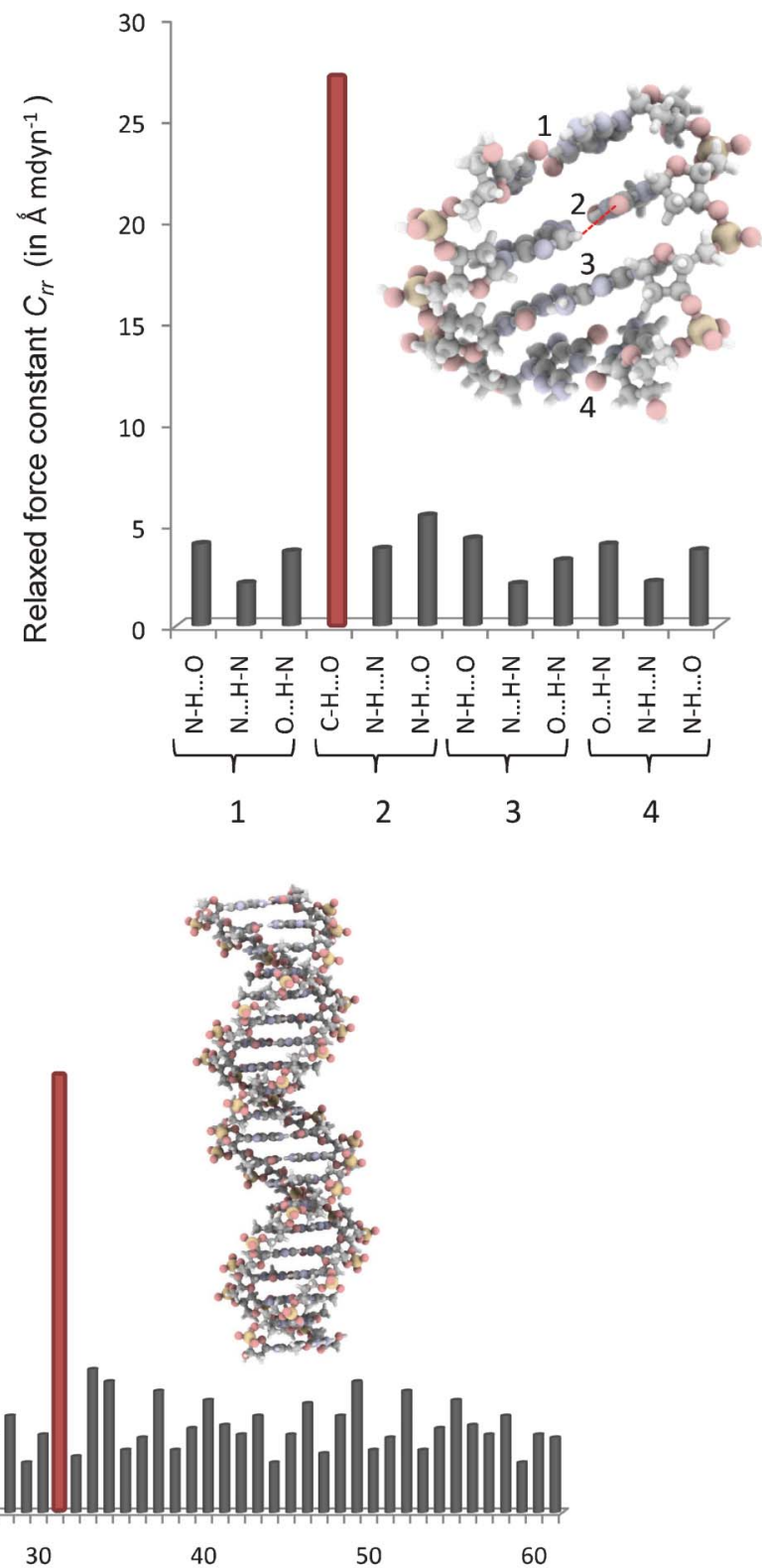

60

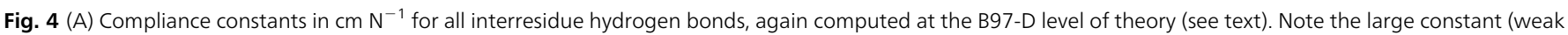

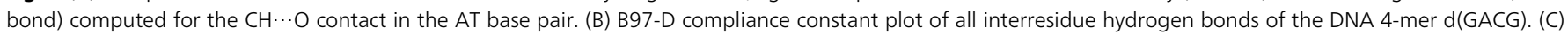

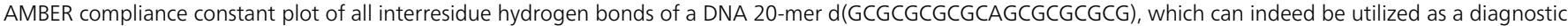
test to identify eminently weak hydrogen bonds. 


\section{Multivalent complexes}

In the second part of our study we now turn to larger molecular systems and more complex interactions. Molecular recognition or biochemical signaling in general is mediated by multivalent binding, where proteins establish recognition complexes providing a multitude of individual soft interactions. It is therefore of great importance to quantify those individual non-covalent bond strengths when it comes to the "design" of tailored artificial receptors. ${ }^{43,44}$ Some years ago we therefore proposed the use of compliance constants for the characterization of soft interactions. ${ }^{26}$ Nevertheless, it was put forward by Pulay and Baker, again based on assumption (2), that "compliance constants (or relaxed force constants) measure the total interaction between fragments, but not individual bonding interactions, and are therefore not useful diagnostics of the strength of weak interatomic interactions". ${ }^{21}$

Several questions arise and a relook at adenine/thymine and guanine/cytosine base pairs might therefore be illuminating. ${ }^{26}$ First of all, if compliance constants measure somehow "the total interaction", why do we see different compliance values for different individual interresidue hydrogen bonds? The fragments, which means the base pairs, are the same for each hydrogen bond. For example, in the case of the AT base pair, a weak $\mathrm{CH} \cdots \mathrm{O}$ bond is neighbored by a strong $\mathrm{NH} \cdots \mathrm{O}$ and a very strong (linear) $\mathrm{NH} \cdots \mathrm{H}$ hydrogen bond. In order to study the robustness of our early results ${ }^{26}$ reconsidering larger and larger molecular surroundings, we now took one step further and computed the full compliance matrix for a model DNA 4-mer d(GACG) at the DFT level of theory (see, Fig. 4A). The starting B-DNA conformer was generated using a rise of $3.3 \AA$ and a twist of 36.0 degrees per base, respectively. The initial strain was released, applying an Amber force field until a gradient of 0.002 was achieved. The resulting structure was submitted to a full quantum chemical optimization followed by calculation of the Cartesian energy second derivatives. Transformation into internal redundant coordinates and inversion was again accomplished by our proposed algorithm $^{29}$ using a modified Moore Penrose strategy. ${ }^{45}$ The results are compiled in Fig. 4.

While the hydrogen bonds connecting GC pairs (1, 3 and 4) retain more or less their elasticity in comparison with the values for the isolated base pairs, ${ }^{26}$ the weak $\mathrm{CH}^{\cdots} \mathrm{O}$ bond in base pair 2 is actually alleviated due to a slight twist of the AT pair in the 4-mer d(GACG). Nevertheless, the main pattern is the same as for the isolated bases pairs: while the central N$\mathrm{H} \cdots \mathrm{N}$ hydrogen bond in the GC base pair is by far the strongest DNA hydrogen bond, the $\mathrm{NH}^{\cdots} \mathrm{O}$ hydrogen bond strength is moderate. This is true for both the AT and the GC base pairs. In the case of the AT pair, the hydrogen bonds are fine tuned by a flimsy but attractive $\mathrm{C}-\mathrm{H} \cdots \mathrm{O}$ interaction.

We finally went another step forward and computed the full compliance matrix for a DNA 20-mer comprising of 19 GC pairs and only one single AT pair in order to further check the robustness of our method for a system comprising of 1258 atoms and 3768 degrees of freedom. Applying the AMBER force field, the initial strain was again released until a gradient of 0.002 was achieved. The resulting structure was submitted to a full force field optimization followed by calculation of the Cartesian energy second derivatives. Transformation into internal redundant coordinates and inversion was again accomplished by our proposed COMPLIANCE algorithm. The results are again compiled in Fig. 4C: While the central $\mathrm{N}-$ $\mathrm{H} \cdots \mathrm{N}$ hydrogen bond in the GC base pairs is still described as the strongest DNA hydrogen bond, the $\mathrm{NH} \cdots \mathrm{O}$ interaction strength is moderate, likewise. The hydrogen bonds connecting GC pairs again retain their relative elasticity in comparison with the values for the isolated base pairs and the DNA 4-mer. Most importantly, the weak $\mathrm{CH}^{\cdots} \mathrm{O}$ bond in base pair $\mathbf{1 1}$ is again easily identifiable, even in a system of 60 interresidue hydrogen bonds.

\section{Conclusions}

All in all, we come to the following conclusions: (1) using compliance constants as bond strength descriptors for soft interactions, the underlying physics is described qualitatively and quantitatively correctly. (2) Compliance constants do not measure the "total interaction of fragments" as asserted sometimes in the literature. This erroneous conclusion was based on oversimplified potential functions. (3) The emergence of a non-zero potential constant for non-neighbor atoms does not preclude the use of compliance constants for the description of next-neighbor interactions. (4) Compliance coupling (off-diagonal) elements describe the relaxation of all other internal coordinates in response to an applied external force. (5) Even in a system containing 60 interresidue hydrogen bonds the compliance matrix retains its local property and might be utilized as a diagnostic test to identify eminently weak hydrogen bonds. An appropriate use of compliance constants for the description of weak bonds might therefore indeed open new paths in the field of soft matter research.

\section{References}

1 R. I. Ainsworth, D. Di Tommaso and N. H. de Leeuw, J. Chem. Phys., 2011, 135, 234513.

2 A. Bailey and N. J. Mosey, J. Chem. Phys., 2012, 136, 044102.

3 A. Espinosa and R. Streubel, Chem.-Eur. J., 2011, 17, 3166.

4 Z. Huang and R. Boulatov, Chemical Society Reviews, 2011, 40.

5 J. H. Brown, V. S. Senthil Kumar, E. O’Neall-Hennessey, L. Reshetnikova, H. Robinson, M. Nguyen-McCarty, A. G. Szent-Györgyi and C. Cohen, Proc. Natl. Acad. Sci. U. S. A., 2010, 108, 114.

6 Several prominent scientists were active in this field at that time: B. I. Swanson, H. S. Rzepa, M. J. S. Dewar, I. H. Williams, in order to name just a few. See B. I. Swanson, J. J. Rafalko, H. S. Rzepa, M. J. S. Dewar and J. Am. Chem. Soc., 1977, 99, 7829.

7 I. H. Williams, Chem. Phys. Lett., 1982, 88, 462. 
8 J. S. Francisco and I. H. Williams, Chem. Phys., 1985, 95, 373.

9 G. von Frantzius, R. Streubel, K. Brandhorst and J. Grunenberg, Organometallics, 2006, 25, 118.

10 P. R. Schreiner, H. P. Reisenauer, J. Romanski and G. Mloston, Angew. Chem., Int. Ed., 2009, 48, 8133.

11 S. Shaik, D. Danovich, W. Wu, P. Su, H. S. Rzepa and P. C. Hiberty, Nat. Chem., 2012, 4, 195.

12 F. Jensen, Introduction to Computational Chemistry, John Wiley \& Sons, 1999, p. 233.

13 J. C. Decius, J. Chem. Phys., 1953, 21, 1121.

14 Carter and Goddard reported that this effect may lead to a variation of the evaluated bond strength by more than 70 kcal $\mathrm{mol}^{-1}$ even for a trivial case like the carbon-carbon double bond: E. A. Carter and W. A. Goddard, III, J. Phys. Chem., 1986, 90, 998. Turning to the heavier elements, the situation may become completely confusing: by reason of a pronounced stabilization of the singlet silylen $\mathrm{SiH}_{2}$, the "strength" of the $\mathrm{Si}=\mathrm{Si}$ double bond in disilene, measured as dissociation energy ( $\left.270 \mathrm{~kJ} \mathrm{~mol}^{-1}\right)$ seems to be "weaker" than a $\mathrm{Si}-\mathrm{Si}$ single bond $310 \mathrm{~kJ} \mathrm{~mol}^{-1}$ ).

15 K. Brandhorst and J. Grunenberg, ChemPhysChem, 2007, 8, 1151.

16 K. Brandhorst and J. Grunenberg, Chem. Soc. Rev., 2008, 37, 1558.

17 A. G. Ponomareva, Y. P. Yurenko, R. O. Zhurakivsky, T. van Mourik and D. M. Hovorun, Phys. Chem. Chem. Phys., 2012, 14, 6787.

18 W. Zou, R. Kalescky, E. Kraka and D. Cremer, J. Chem. Phys., 2012, 137, 084114.

19 Z. Konkoli and D. Cremer, Int. J. Quantum Chem., 1998, 67, 1.

20 W. T. Taylor and K. S. Pitzer, J. Res. Natl. Bur. Stand., 1947, $38,1$.

21 J. Baker and P. Pulay, J. Am. Chem. Soc., 2006, 128, 11324.

22 J. Baker, J. Chem. Phys., 2006, 125, 014103.

23 Confusion of perceptions like "bond strength" or "bond order" may also led to misinterpretations concerning the suitability of compliance constants as bond descriptors. See, for example: M. V. Madhav and S. Manogaran, J. Chem. Phys., 2009, 131, 174112.

24 M. Lein, Coord. Chem. Rev., 2009, 253, 625.

25 I. G. Kaplan, Intermolecular Interactions: Physical Picture, Computational Methods and Model Potentials, John Wiley and Sons Ltd, 2006.

26 J. Grunenberg, J. Am. Chem. Soc., 2004, 126, 16310.

27 S. S. Zhu, H. Staats, K. Brandhorst, J. Grunenberg, F. Gruppi, E. Dalcanale, A. Lützen, K. Rissanen and C. A. Schalley, Angew. Chem., Int. Ed., 2008, 47, 788.

28 Thomas Kleine-Ostmann, R. Wilk, F. Rutz, M. Koch, H. Niemann, B. Güttler, K. Brandhorst and J. Grunenberg, ChemPhysChem, 2008, 9, 544.

29 K. Brandhorst and J. Grunenberg, J. Chem. Phys., 2010, 132, 184101.
30 M. Preller, J. Grunenberg, V. P. Bulychev and M. O. Bulanin, J. Chem. Phys., 2011, 134, 174302.

31 R. R. Lucchese, J. W. Bevan and F. J. Lovas, Chem. Phys. Lett., 2004, 398, 544.

32 J. A. Pople, M. Head-Gordon and K. Raghavachari, J. Chem. Phys., 1987, 87, 5968.

33 H. Dunning Thom, Jr., J. Chem. Phys., 1989, 90, 1007.

34 K. A. Peterson, D. Figgen, M. Dolg and H. Stoll, J. Chem. Phys., 2007, 126, 124101.

35 K. A. Peterson, B. C. Shepler, D. Figgen and H. Stoll, J. Phys. Chem. A, 2006, 110, 13877.

36 G. W. T. M. J. Frisch, H. B. Schlegel, G. E. Scuseria, M. A. Robb, J. R. Cheeseman, G. Scalmani, V. Barone, B. Mennucci, G. A. Petersson, H. Nakatsuji, M. Caricato, X. Li, H. P. Hratchian, A. F. Izmaylov, J. Bloino, G. Zheng, J. L. Sonnenberg, M. Hada, M. Ehara, K. Toyota, R. Fukuda, J. Hasegawa, M. Ishida, T. Nakajima, Y. Honda, O. Kitao, H. Nakai, T. Vreven, J. A. Montgomery, Jr., J. E. Peralta, F. Ogliaro, M. Bearpark, J. J. Heyd, E. Brothers, K. N. Kudin, V. N. Staroverov, R. Kobayashi, J. Normand, K. Raghavachari, A. Rendell, J. C. Burant, S. S. Iyengar, J. Tomasi, M. Cossi, N. Rega, J. M. Millam, M. Klene, J. E. Knox, J. B. Cross, V. Bakken, C. Adamo, J. Jaramillo, R. Gomperts, R. E. Stratmann, O. Yazyev, A. J. Austin, R. Cammi, C. Pomelli, J. W. Ochterski, R. L. Martin, K. Morokuma, V. G. Zakrzewski, G. A. Voth, P. Salvador, J. J. Dannenberg, S. Dapprich, A. D. Daniels, Ö. Farkas, J. B. Foresman, J. V. Ortiz, J. Cioslowski and D. J. Fox, in 'Gaussian 09, Revision A.1, Wallingford CT, 2009.

37 K. L. Schuchardt, B. T. Didier, T. Elsethagen, L. Sun, V. Gurumoorthi, J. Chase, J. Li and T. L. Windus, J. Chem. Inf. Model., 2007, 47, 1045.

38 In fact, this reasoning mixes a pre quantum concept of bond order (Lewis structure) and valence theory, on the one side and the Hellmann-Feynman forces (the negative of the gradient of the potential energy of interaction between the two nuclei) on the other side. See: R. F. W. Bader, Phys. Rev. B: Condens. Matter, 2000, 61, 7795.

39 We propose the term "compliance modes" in order to strictly distinguish them from normalmodes. The modes can be visualized using our Compliance software package: Brandhorst and J. Grunenbergin 'Compliance 3.0.1', 2009.

40 N. Bjerrum, Verh. d. D. Phys. Ges., 1914, 16, 737.

41 E. B. Wilson, J. C. Decius and P. C. Cross, 'Molecular Vibrations', Dover, 1989, page173.

42 J. Emsley, Chem. Soc. Rev., 1980, 9, 91.

43 S. Zhu, H. Staats, K. Brandhorst, J. Grunenberg, F. Gruppi, E. Dalcanale, A. Lützen, K. Rissanen and C. H. A. Schalley, Angew. Chem., Int. Ed., 2008, 47, 788.

44 J. Grunenberg, Phys. Chem. Chem. Phys., 2011, 13, 10136.

45 R. A. Penrose, Proc. Camb. Phil. Soc., 1955, 51, 406.

46 R. Bast, A. Heßelmann, P. Sałek, T. Helgaker and T. Saue, ChemPhysChem, 2008, 9, 445. 